Research Article

\title{
Intravoxel Incoherent Motion (IVIM) Diffusion-Weighted Imaging (DWI) in Patients with Liver Dysfunction of Chronic Viral Hepatitis: Segmental Heterogeneity and Relationship with Child-Turcotte-Pugh Class at 3 Tesla
}

\author{
Lei Ding (iD, ${ }^{1}$ Lianxiang Xiao, ${ }^{2}$ Xiangtao Lin, ${ }^{2}$ Chunmei Xiong, ${ }^{3}$ Lingbo Lin, ${ }^{4}$ \\ and Shijun Chen $\mathbb{1}^{4}$ \\ ${ }^{1}$ Shandong University, Department of Infectious Diseases, Jinan Central Hospital Affiliated to Shandong University, Jinan, \\ 250021 Shandong Province, China \\ ${ }^{2}$ Shandong Medical Imaging Research Institute, Jinan, 250021 Shandong Province, China \\ ${ }^{3}$ Shandong University, Jinan Infectious Diseases Hospital, Jinan, 250021 Shandong Province, China \\ ${ }^{4}$ Jinan Infectious Diseases Hospital, Jinan, 250021 Shandong Province, China
}

Correspondence should be addressed to Shijun Chen; csj7516@sina.com

Received 5 July 2018; Accepted 19 September 2018; Published 16 December 2018

Academic Editor: Amosy M'Koma Copyright $\odot 2018$ Lei Ding et al. This is an open access article distributed under the Creative Commons Attribution License, which
permits unrestricted use, distribution, and reproduction in any medium, provided the original work is properly cited.

\begin{abstract}
Background. Few studies focused on the region of interest- (ROI-) related heterogeneity of liver intravoxel incoherent motion (IVIM) diffusion-weighted imaging (DWI). The aim of the study was to evaluate the differences of liver IVIM parameters among liver segments in cirrhotic livers (chronic viral hepatitis). Material and Methods. This was a retrospective study of 82 consecutive patients with chronic liver disease who underwent MRI examination at the Jinan Infectious Diseases Hospital between January 2015 and December 2016. IVIM DWI (seven different $b$ values) was performed on a Siemens 3.0-T MRI scanner. Pure molecular diffusion $(D)$, pseudodiffusion $\left(D^{*}\right)$, and perfusion fraction $(f)$ in different liver segments were evaluated. Results. $f, \mathrm{D}$, and $D^{*}$ were different among the liver segments (all $p<0.05$ ), indicating heterogeneity in IVIM parameters among liver segments. $f$ was consistently higher in Child-Turcotte-Pugh (CTP) class A compared with CTP class $\mathrm{B}+\mathrm{C}(p<0.01) . D$ and $D^{*}$ were higher in CTP class A compared with CTP class B + C $(p<0.05)$. In patients with mean $f$ value of $>0.29$, the AUC was 0.88 (95\% CI: $0.81-0.96)$, with $86.8 \%$ sensitivity and $81.8 \%$ specificity for predicting CTP class A from CTP class B + C. Conclusion. Liver IVIM could be a promising method for classifying the severity of segmental liver dysfunction of chronic viral hepatitis as evaluated by the CTP class, which provides a noninvasive alternative for evaluating segmental liver dysfunction with accurate selection of ROIs. Potentially it can be used to monitor the progression of CLD and LC in the future.
\end{abstract}

\section{Introduction}

Liver function estimation plays an essential role in predicting the prognosis of patients with chronic liver disease (CLD) or liver cirrhosis (LC), both of which ultimately lead to liver failure. For patients within a background of CLD or LC with or without hepatocellular carcinoma (HCC), assessment of liver function is also an integral part of the therapeutic decision making and can help physicians make the appropriate treatment decision [1,2]. In clinical practice, indocyanine green clearance test, elastography, and clinical scoring systems such as the Child-Turcotte-Pugh (CTP) or Model for End-Stage Liver Disease (MELD) scores are used to evaluate whole liver function. CTP score is a widely used and validated predictor of long-term survival in CLD and LC, and patients are grouped into class $\mathrm{A}, \mathrm{B}$, and $\mathrm{C}$ according to the total score of $5-6,7-9$, and $10-15$, respectively [3]. 
Magnetic resonance imaging (MRI) using gadoxetic acid and intravoxel incoherent motion (IVIM) diffusionweighted imaging (DWI) has recently shown a potential for the evaluation of segmental liver dysfunction [4-8]. IVIM DWI, which was initially described by Le Bihan and Turner [9] in brain imaging, has the potential to measure both true molecular diffusion and the incoherent motion of water molecules in the capillary network. By using the IVIM model and multiple sufficiently low $b$ values $\left(<200 \mathrm{~mm}^{2} / \mathrm{sec}\right)$, not only can pure diffusion characteristics $(D)$ be separated from pseudodiffusion caused by microscopic circulation in tissue, but perfusion characteristics (pseudodiffusion coefficient $\left(D^{*}\right)$ ) and their proportion (perfusion fraction $(f)$ ) can also be derived [10-12]. Using IVIM DWI, perfusion and diffusion factors can be separated [2]. Nevertheless, for CLD or LC, the perfusion and microscopic phenomena of liver are heterogeneous due to progressive increase in connective tissue and reduced liver perfusion $[4,9]$. Indeed, the variations associated with the acquisition sites of shear-wave elastography for evaluating liver fibrosis stage have been proven by Samir et al. [13]. Thus, the IVIM parameters may vary among different segments due to the regions of interest (ROIs) location. Recent studies of liver dysfunction or fibrosis evaluated by IVIM refer to different ROIs location and boundary from a single segment to the whole liver [7, 14-18].

Few studies focused on the ROI-related heterogeneity of liver IVIM parameters. Therefore, the aim of this study was to evaluate the differences of liver IVIM parameters among liver segments in cirrhotic livers caused by chronic viral hepatitis and determine the relationships between IVIM measurements and liver dysfunction assessment according to the CTP scoring system.

\section{Material and Methods}

2.1. Study Design. This was a retrospective study of 82 consecutive patients with CLD who underwent MRI examination at the Department of Radiology of Jinan Infectious Diseases Hospital between January 2015 and December 2016. The study was approved by the ethics committee of Jinan Infectious Diseases Hospital. The need for individual consent was waived because of the retrospective nature of the study.

2.2. Patients. For those patients, MRI examination was primarily performed to observe the morphological changes of liver and the secondary changes of hepatitis/cirrhosis, such as nodules and ascites, and to exclude HCC. The inclusion criteria were (1) >18 years of age, (2) chronic infection with hepatitis $B$ virus (HBV) or hepatitis $C$ virus (HCV), and (3) IVIM was performed. The exclusion criteria were (1) previously received local treatment for liver disease, (2) unable to complete the entire MR imaging examination, (3) other diffuse liver disease (primary sclerosing cholangitis, primary biliary cirrhosis, hepatic adipose infiltration, etc.), (4) HCC confirmed by MRI, cyst, and hemangioma $1 \mathrm{~cm}$ or greater in diameter

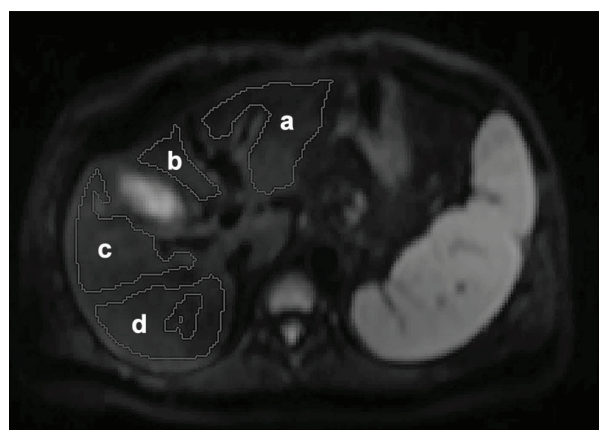

FIgURE 1: Regions of interest (ROIs) drawing for intravoxel incoherent motion diffusion-weighted imaging measurements. $a=$ extra segment of the left lobe; $b=$ medial segment of the left lobe; $c=$ anterior segment of the right lobe; $d=$ posterior segment of the right lobe.

confirmed by MRI (other tumors or tumor-like lesions were not found for those patients), (5) portal vein emboli, or (6) alcohol abuse or alcoholic cirrhosis.

2.3. Biochemical Tests and Liver Function. All patients underwent serological tests in the same laboratory within 1 week before or after MRI. The severity of liver disease was estimated by the CTP scoring system.

2.4. MRI Examination and IVIM Parameters. All patients were instructed to fast and abstain from food and water overnight prior to MRI examination. MRI was performed using a 3.0-T scanner (Magnetom Verio, Siemens Healthcare, Erlangen, Germany). A body coil served as the transmitter and a 6-element spine matrix coil in combination with the body matrix were used as the receiver. At first, coronal T2weighted imaging (half-Fourier acquisition single-shot turbo spin-echo (HASTE), repetition time (TR)/echo time (TE) $1400 / 86 \mathrm{~ms}$, flip angle 10 , matrix $512 \times 512$, field of view $400 \times 400 \mathrm{~mm}$, slice thickness $5 \mathrm{~mm}, 20 \%$ gap, 30 slices) was performed.

The transverse MRI protocol included liver dome scout-triggered transverse T2-weighted turbo spin-echo sequence (TR/TE 4251/105 ms, matrix 560, field of view $400 \times 400 \mathrm{~mm}$, slice thickness $5 \mathrm{~mm}, 20 \%$ gap, 30 slices), and 3D in-phase and out-of-phase breath-hold fast spoiled gradient-echo imaging (TR/TE, 4.0/2.5 and $1.2 \mathrm{~ms}$, flip angle 10 , matrix $512 \times 512$, field of view $450 \times 390 \mathrm{~mm}$, slice thickness $3 \mathrm{~mm}, 72$ slices).

Free-breathing, IVIM DWI was performed using a single-shot spin-echo echo planar sequence (SE-EPI), with gradient reversal fat suppression (TR/TE 6500/67 ms, echo spacing $0.52 \mathrm{~ms}$, FOV $400 \times 262 \mathrm{~mm}$, using $7 b$ values of 0 , $50,100,150,200,400$, and $800 \mathrm{~s} / \mathrm{mm}^{2}$ ).

2.5. Image Analysis. Postprocessing of the IVIM data were performed by using the MITK diffusion software (developed by the German Cancer Research Center, Division of Medical and Biological Informatics, Heidelberg, Germany) to acquire IVIM parameters of $f, D$, and $D^{*}$. For the liver parenchyma, four irregular ROIs (designed to carefully 


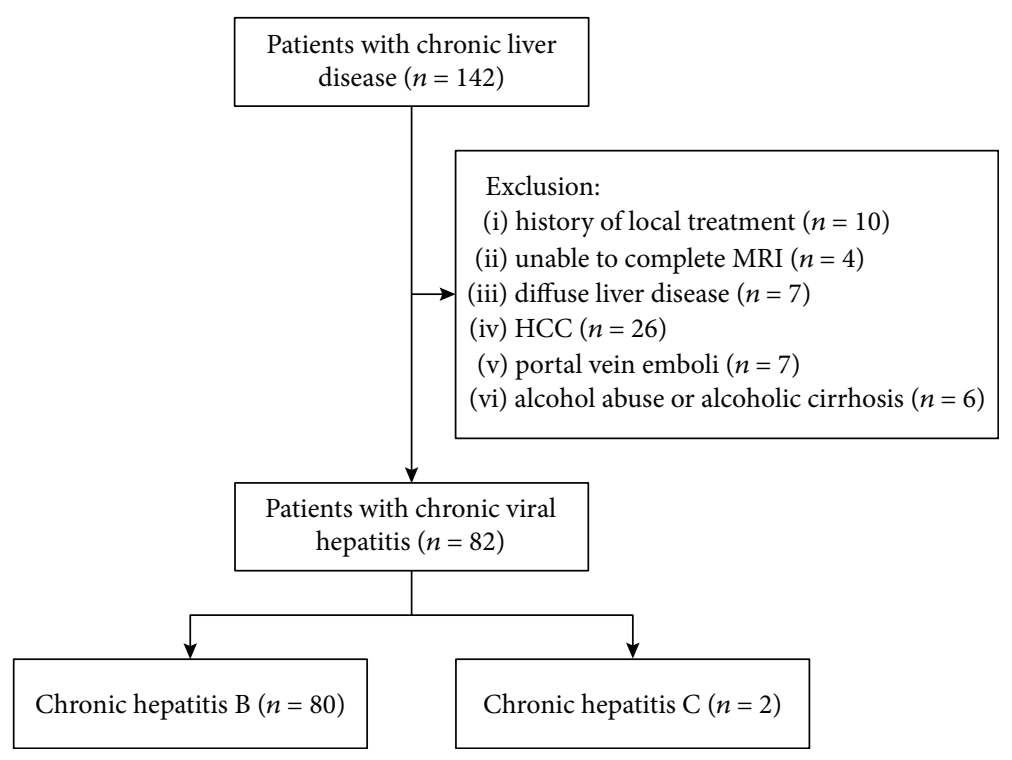

Figure 2: Patient flowchart.

preserve at least $5 \mathrm{~mm}$ to the edge of the liver, including whole segments as large as possible and excluding visible vessels, focal hepatic lesions such as cyst and hemangioma, or imaging artifacts) were placed by choosing different levels of the liver (slices near the visceral and diaphragmatic surfaces were discarded to eliminate intestinal gas and respiratory motion artifacts). The ROIs were manually drawn in the extra segments of the left lobe (EL), medial segments of the left lobe (ML), anterior segments of the right lobe (AR), and posterior segments of the right lobe (PR) (Figure 1). All ROIs were positioned on DWI with $b$ values of 50 by two radiologists, one with 15 years (XTL) and the other with 7 years (LXX) of experience in abdominal MRI. The two radiologists were blind to the clinical characteristics of the patients. Interobserver agreement for all IVIM parameters was excellent, with Cronbach's $\alpha$ of 0.951 for $f, 0.876$ for $D$, and 0.861 for $D^{*}$.

For each liver segment, IVIM parameters $\left(f, D\right.$, and $\left.D^{*}\right)$ were calculated by the average of measured value at 6-15 different level of transverse liver sections. The average $f, D$, and $D^{*}$ values of the four liver segments were taken as the whole liver IVIM parameters.

2.6. Statistical Analysis. Continuous data were tested for normal distribution using the Kolmogorov-Smirnov test. The IVIM parameters were expressed as mean \pm standard deviations. One-way ANOVA with the LSD post hoc test was used to evaluate IVIM parameters among different liver segments. IVIM parameters between the CTP class A group and the CTP class $\mathrm{B}+\mathrm{C}$ group were compared using the Student $t$-test. The receiver operator characteristic curve (ROC) was used to compare the ability of $f, D$, and $D^{*}$ values in discriminating patients with CTP class $A$ and CTP class $\mathrm{B}+\mathrm{C}$. Multiple linear regression analysis was used to evaluate the correlations between the IVIM
TABle 1: Clinical characteristics of 82 patients with chronic viral hepatitis evaluated using IVIM MRI.

\begin{tabular}{lcc}
\hline Characteristics & Value (mean, SD) & Median (range) \\
\hline Age $($ years $)$ & $52.7 \pm 11.9$ & $51(27-77)$ \\
Male/female, $n(\%)$ & $51(62.2) / 31(37.8)$ & \\
BMI $\left(\mathrm{kg} / \mathrm{m}^{2}\right)$ & $24.1 \pm 2.8$ & $24.2(17.9-24.3)$ \\
Hepatitis B/hepatitis C & $80 / 2$ & \\
INR & $1.3 \pm 0.3$ & $1.26(0.95-2.9)$ \\
ALT (IU/L) & $81.4(141)$ & $40.5(13-1005)$ \\
AST (IU/L) & $92.7 \pm 105$ & $54.5(19-691)$ \\
ALB (g/L) & $34.4 \pm 6.5$ & $34(17-54.7)$ \\
TBIL (mmol/L) & $40.9 \pm 62.1$ & $21.7(4.9-375.4)$ \\
AFP (ng/mL) & $119.4 \pm 250.6$ & $14.9(0.84->2000)$ \\
CTP score, mean or n & $6.9 \pm 1.4$ & \\
CTP class A (5 6) & 40 & \\
5 & 24 & \\
6 & 16 & \\
CTP class B (7 9) & 40 & \\
7 & 29 & \\
8 & 5 & \\
9 & 2 & \\
CTP class C (10) & 6 & \\
\hline
\end{tabular}

BMI: body mass index; INR: international normalized ratio; ALT: alanine transaminase; AST: aspartate transaminase; ALB: albumin; TBIL: total bilirubin; AFP: $\alpha$-fetoprotein.

parameters and the CTP scores. All statistical analyses were performed using SPSS 21.0 for Windows (IBM, Armonk, NY, USA). Two-sided $p$ values $<0.05$ were considered statistically significant. 


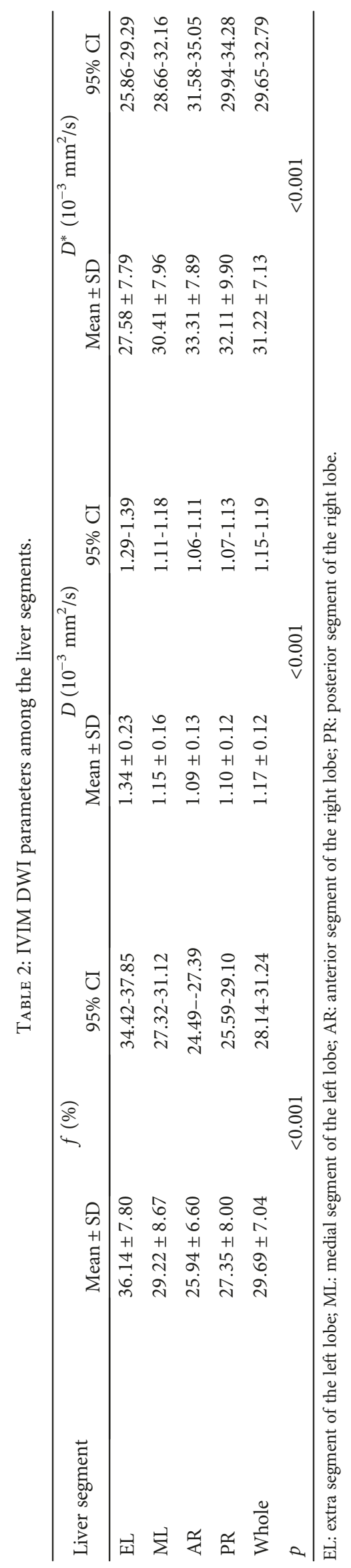



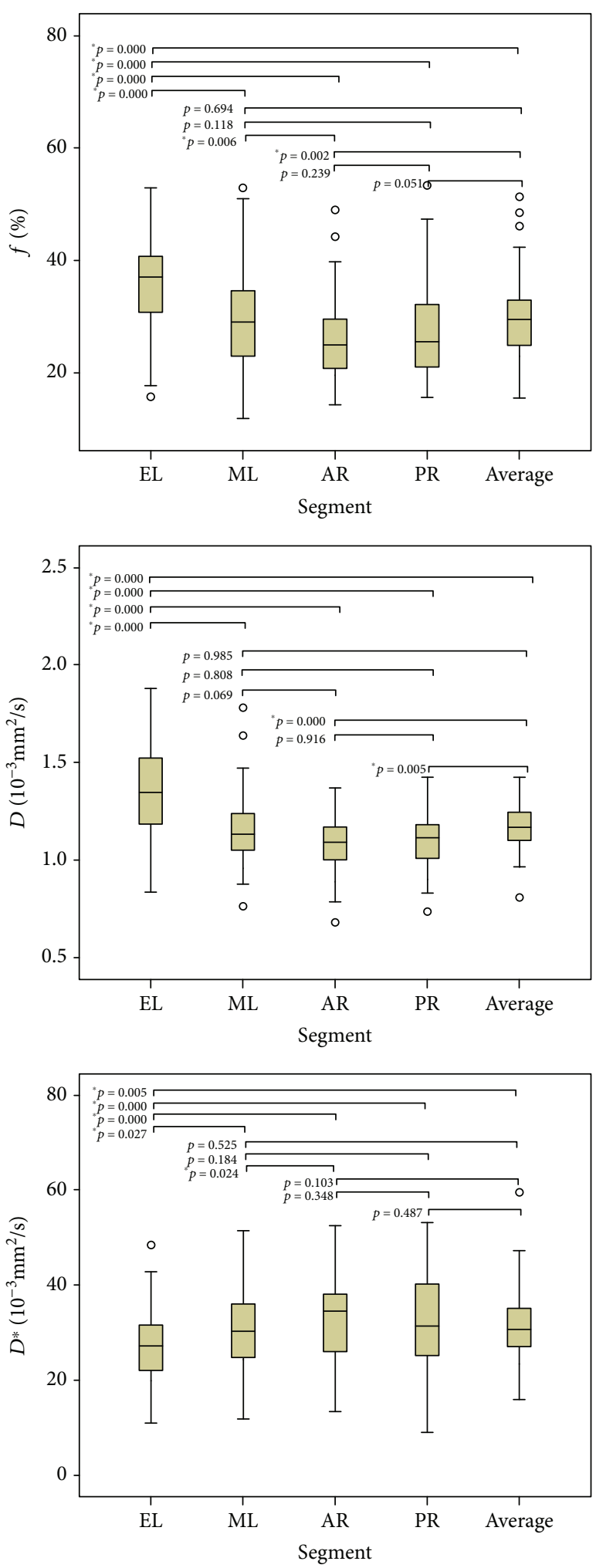

Figure 3: Box plots of $f, D$, and $D^{*}$ in different liver segments.

\section{Results}

3.1. Patients. A total of 142 patients with CLD were considered for inclusion. Patients were excluded if they had previously received a local treatment for liver disease $(n=10)$, if they were unable to complete the entire MRI examination $(n=4)$, if they had other diffuse liver disease $(n=7)$, if they had HCC confirmed by MRI $(n=26)$, if they had portal vein emboli $(n=7)$, or if they had alcohol abuse or alcoholic cirrhosis $(n=6)$. As shown in Figure 2, 82 patients (age range 24-77 years) with chronic viral hepatitis were included in this study. The clinical characteristics of these patients are listed in Table 1.

3.2. IVIM Parameters in Different Location. The IVIM parameters of the different liver segments and of the whole liver were normally distributed $(p=0.310-0.899$, Kolmogorov-Smirnov test). The descriptive statistics are presented in Table 2. Figure 3 shows that $f, D$, and $D^{*}$ were significantly different among the liver segments, indicating heterogeneity in IVIM parameters among different liver segments.

3.3. Relationship between IVIM Parameters and CTP Class. Table 3 shows $f, D$, and $D^{*}$ of different liver segments according to the CTP class. $f$ was consistently higher in CTP class A than in CTP class B $+\mathrm{C}($ all $p<0.01)$. D was higher in CTP class A compared with CTP class B $+C$ in the EL, PR, and whole liver (all $p<0.05) . D^{*}$ was higher in CTP class A compared with CTP class B $+C$ in the EL, ML, $\mathrm{PR}$, and whole liver (all $p<0.05$ ).

3.4. ROC Analysis. As shown in Figure 4, the area under the ROC curves (AUC) for $f, D$, and $D^{*}$ value were statistically significant. In patients with mean $f$ value of $>0.29$, the AUC was 0.88 (95\% CI: 0.81-0.96), which was the highest of the three IVIM parameter, leading to $86.8 \%$ sensitivity and $81.8 \%$ specificity for predicting CTP class A from CTP class B $+\mathrm{C}$ (Table 4$)$.

3.5. Multivariate Analysis. Multiple linear regression analysis showed that in viral hepatitis patients, $f(p<0.001)$ and $D$ $(p=0.038)$ were independently associated with the CTP class, while $D^{*}$ was not associated $(p=0.451)$.

3.6. Typical Cases. Figures 5 and 6 present two typical cases. Both cases were relatively stable. The patient presented in Figure 5 was CTP class A. $f$ EL was $0.35, f$ ML was $0.24, f$ AR was 0.29, $f$ PR was 0.43, and whole liver $f$ was 0.33 . According to the critical value of 0.29 in the present study, the liver function evaluated by $f$ value showed that the whole liver $f$ value was consistent with CTP class A, but if a single $\mathrm{ROI}$ is placed in the left inner lobe or right anterior lobe, or if using multiple ROIs, a false positive result would be obtained and the patient would be identified as a CTP class $\mathrm{B}+\mathrm{C}$. In a similar manner, in Figure 6 , the patient was CTP class B. $f$ EL was $0.25, f \mathrm{ML}$ was $0.29, f \mathrm{AR}$ was $0.22, f \mathrm{PR}$ was 0.37 , and whole liver $f$ was 0.28 . Nevertheless, if the ROI was placed in the right posterior lobe, then the patient would be determined as CTP class A. These two typical cases clearly demonstrate the importance of liver heterogeneity and liver function local assessment in cirrhotic patients. 


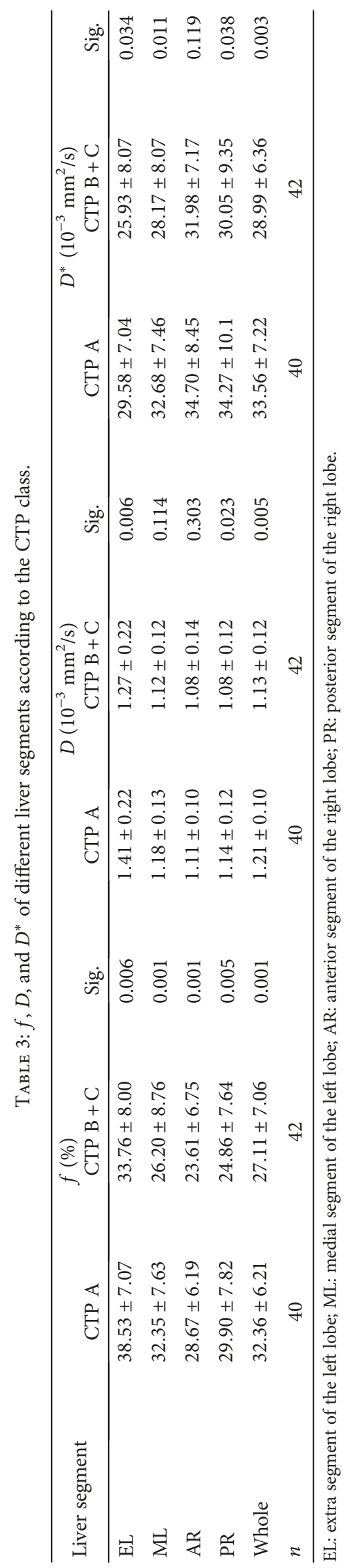



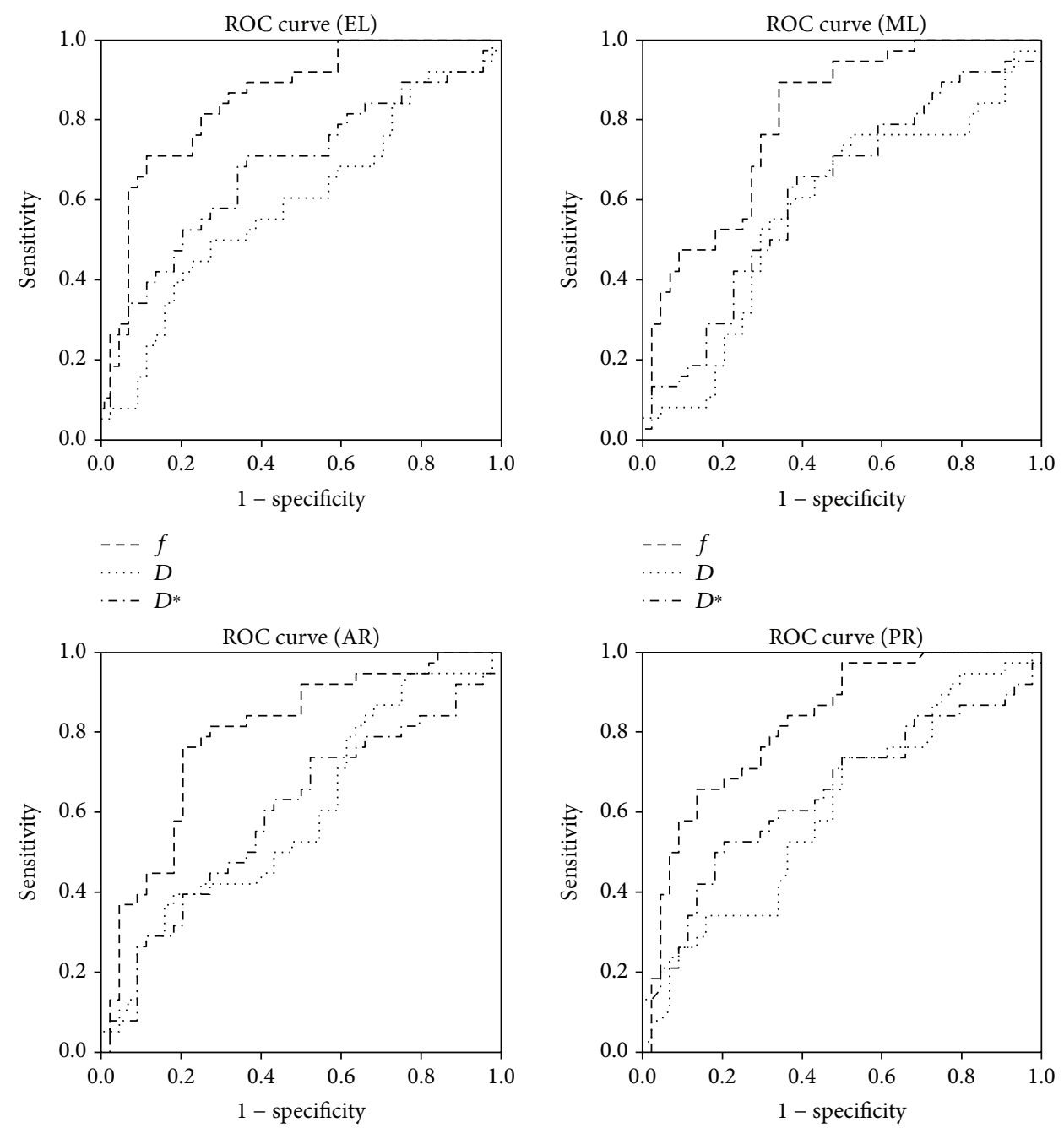

$$
\begin{array}{ll}
--- & f \\
\cdots \cdots & D \\
-- & D^{*}
\end{array}
$$$$
---f
$$$$
\text { …. } D
$$

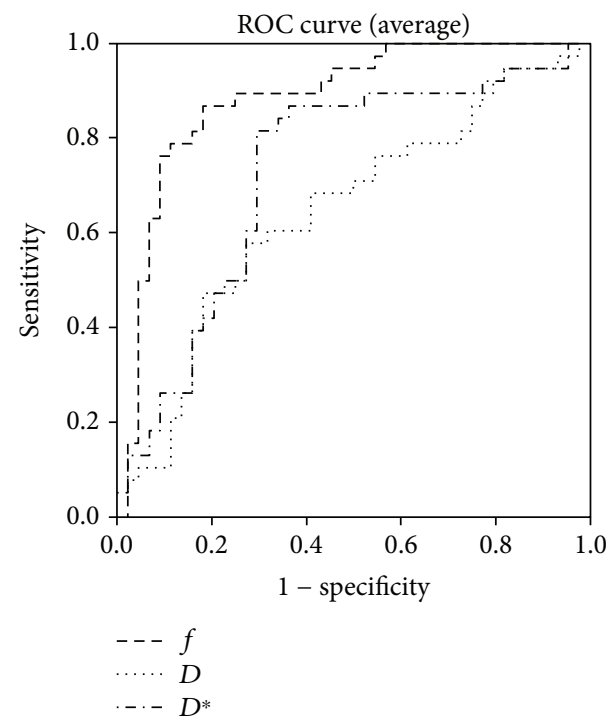

FIGURE 4: Receiver operating characteristic curves comparing $f, D$, and $D^{*}$ in different segments as predictors of CTP class (A vs. B + C). 
TABle 4: ROC curves data of $f, D$, and $D^{*}$ for distinguishing CTP class A vs. B + C.

\begin{tabular}{lccc}
\hline Segment & $f(p$ value $)$ & $\begin{array}{c}\text { AUC } \\
\text { EL value })\end{array}$ & $D^{*}(p$ value $)$ \\
\hline ML & $0.85(<0.001)$ & $0.59(0.158)$ & $0.68(0.005)$ \\
AR & $0.80(<0.001)$ & $0.58(0.234)$ & $0.62(0.062)$ \\
PR & $0.79(<0.001)$ & $0.59(0.142)$ & $0.60(0.139)$ \\
Whole liver & $0.83(<0.001)$ & $0.61(0.099)$ & $0.65(0.021)$ \\
\hline
\end{tabular}

EL: extra segment of the left lobe; ML: medial segment of the left lobe; AR: anterior segment of the right lobe; PR: posterior segment of the right lobe.

\section{Discussion}

The present study revealed a significant variability of IVIM parameters among different liver segments. There were statistically significant higher $f$ and $D$ values, but lower $D^{*}$ values in EL compared with the other segments, as supported by a study by Dijkstra et al. [5]. The heterogeneity of $f$ values was partly similar to that observed in previous studies $[4,5]$. Nevertheless, the present study suggests location dependency in all IVIM parameters including the microperfusion component $D^{*}$ and the pure molecular diffusion component $D$. For the heterogeneity of $D^{*}$, the present study is consistent with the study by Dijkstra et al. [5], but both studies contradict Luciani et al. [4]. A number of studies may be responsible for these differences, including the types of pathologies, ethnic groups, and genetics. In addition, the range observed in the present study was lower than what they reported [4]. This may reflect the use of different $b$ values calculation methods since we chose the three parameters fit model on the MITK diffusion workstation. Significant heterogeneity of $D$ between segments was not observed in these two studies, which could be due to the relatively small number of patients or mild changes in the microenvironment in healthy individuals.

Furthermore, a previous study showed obvious variation, and a large range of $f$ values, $D$ values, and $D^{*}$ values for F0 stage liver tissue [2]. It is well accepted that liver cirrhosis is associated with reduced liver perfusion, particularly with reduced portal flow [19-21]. In an experimental study on rats using perfusion computed tomography (CT), the relative blood flow in the left lobe was $17 \%$ higher than in the right lobe of the liver [22]. This is also supported by Su et al. [23] in a study of hepatic perfusion by dual-source CT. They found that the hepatic perfusion index (HPI) was significantly higher in segment 3 (extra left lobe) than in segments 5 to 8 (right lobe) and suggested that this might be related to the anatomy of the liver vessels. This is supported by the compensatory increase of the left lobe in liver cirrhosis.

We believe that besides the histological changes such as fat and iron content and technical difference such as the choice of $b$ values and cardiac or respiratory artifacts, the acquisition site of ROIs also has an important influence explaining, at least in part, the large range of reported IVIM parameters. These issues add to the heterogeneity observed among liver segments, further complicating the interpretation of the results.

Reduced liver perfusion and progressive increased connective tissue are considered to be the possible mechanisms that could underlie the reduction of IVIM parameters in CLD and liver cancer $[24,25]$. The $D$ values reflect both intra- and extracellular molecular diffusion, while $D^{*}$ and $f$ values reflect the microcirculation. It has been reported that $f$ values are decreased with the increasing severity of the necroinflammatory activity [4, 26-33]. Therefore, IVIM parameter changes may reflect not only the fibrosis degree and perfusion changes but also the hepatitis activity such as inflammatory infiltration, hepatic cell edema, and cholestasis. In the present study, the $f, D$, and $D^{*}$ values were all decreased with increasing CTP score, which is supported by a previous study by Zhang et al. [34]. According to the multiple linear regression analysis, the $f$ and $D$ values were independently associated with the CTP score, but not $D^{*}$. This could partially be because $D^{*}$ is not a well reproducible measure influenced by liver fibrosis [35]. A moderate relation was found between the average $f$ value and CTP score, and a mild relation between either average $D$ value or CTP score. The IVIM parameters of CTP class A were significantly higher than that of CTP class $B+C$, yet there was only mild to moderate correlation between the IVIM parameters and CTP score. This can be partially due to the distribution of the patients, mostly CTP score of 5 to 7 , as observed in most patients of the present study. Furthermore, the $D$ values were influenced by confounders such as fibrosis, fat, and iron, which commonly coexist with liver disease.

Based on previous studies and ours, IVIM DWI could be a quick and repeatable noninvasive MR modality that enables qualitative and quantitative evaluation of tissue diffusivity. It could potentially become a reliable imaging modality to quantify changes in CLD. One of the advantage of IVIM DWI is that it can be integrated into routine abdominal MRI sequences. Compared with the use of gadolinium chelates, there is no restriction of abnormal renal function, as the impaired renal function leads to increased hepatobiliary excretion after injection of $\mathrm{Gd}$ EOB-DTPA [36, 37].

The present study has several limitations. First, histopathological confirmation of liver fibrosis stage was not performed. Secondly, there was no patient with normal liver as a negative control group. In addition, there was only a few patients with high CTP score. Nevertheless, the changes of IVIM parameters in the CTP A group and the CTP B + C group still reflected the tendency of negative correlation with liver dysfunction. In addition, because of software limitations on the MRI system, it was not possible to acquire any data of $D^{*}$ between $b=0$ and $50 \mathrm{~s} / \mathrm{mm}^{2}$, which may result in the relative lower $D^{*}$ measurement in the present study as well as in others' $[5-7,38-41]$. Although the obvious variation and poor 

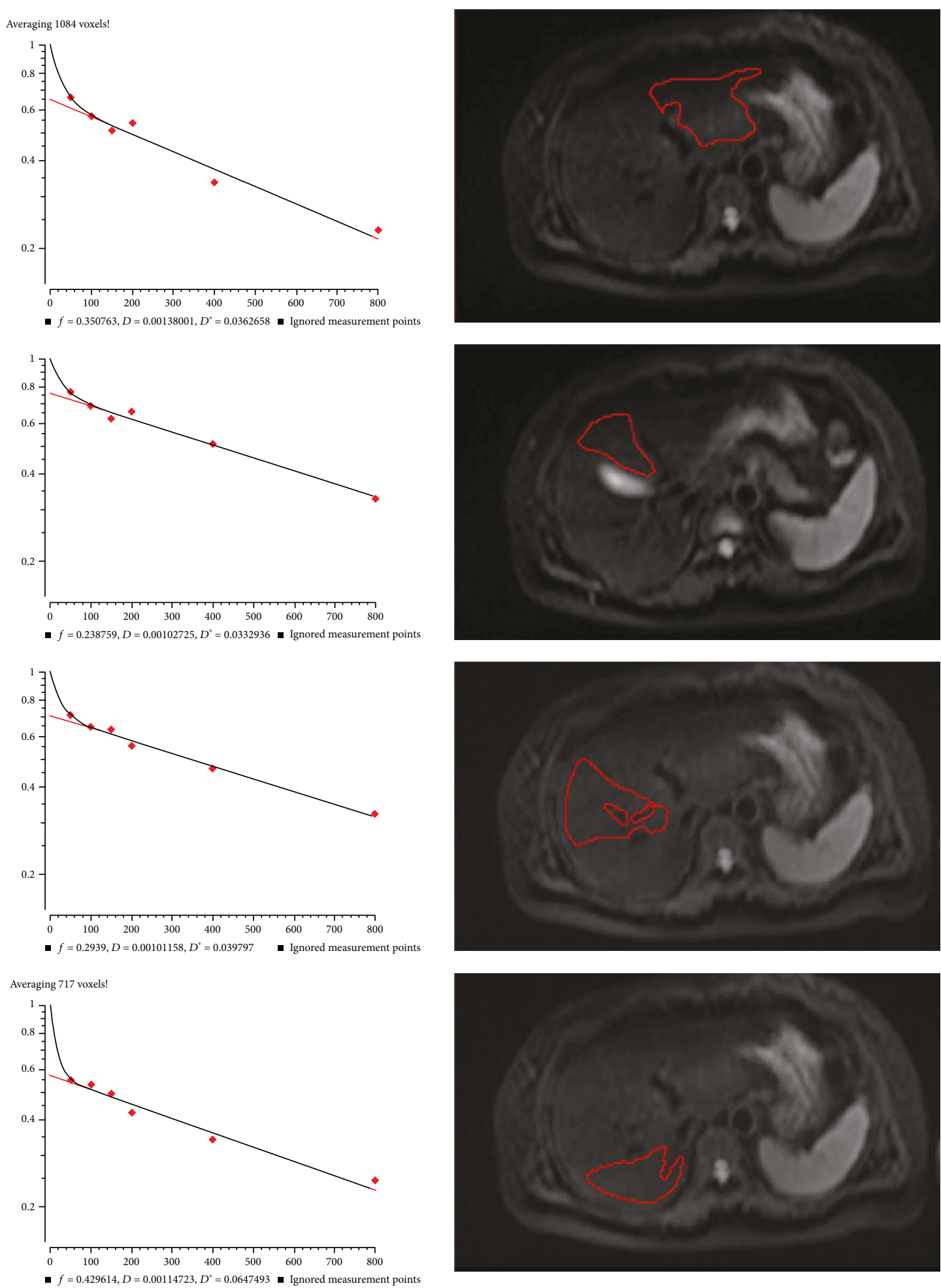

FIGURE 5: IVIM measurements in different liver segments in a 52-year-old female with chronic hepatitis B, CTP score of 6 (class A).

reproducibility of IVIM parameters were doubted by some study [35], it still showed a reasonable potential for quantifying CLD. Among the most influential factors, segmental dependency-related heterogeneity may be underestimated in previous studies.

\section{Conclusions}

IVIM DWI imaging of the liver is a promising modality for classifying the severity of liver dysfunction of chronic viral hepatitis as evaluated by CTP class. It provides a noninvasive 

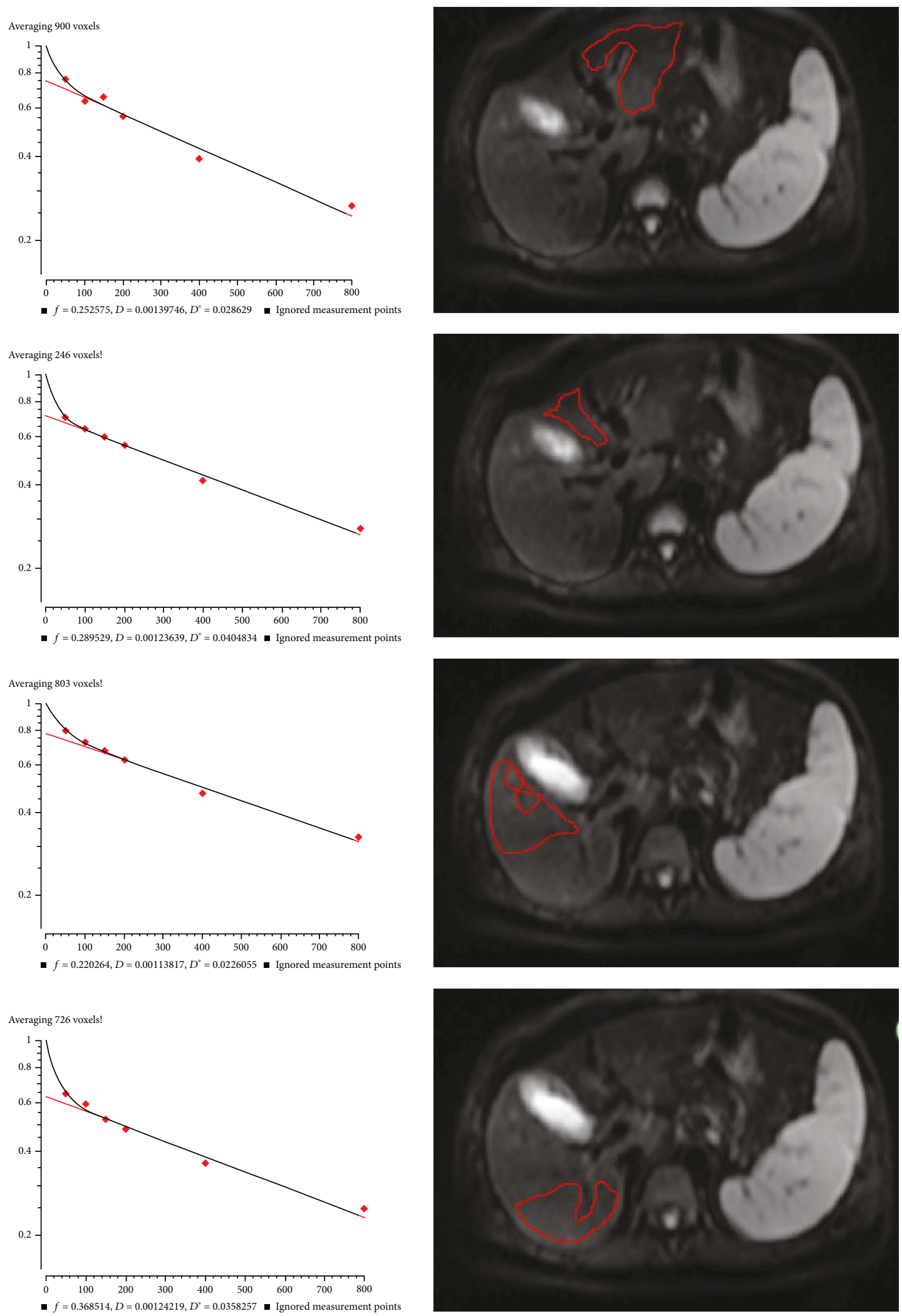

FIGURE 6: IVIM measurements in different liver segment in a 67-year-old female with chronic hepatitis B, CTP score of 9 (class B).

alternative for evaluating segmental liver dysfunction. Clinically, we can potentially use IVIM to monitor the progression of CLD and LC in the future. The heterogeneity of IVIM measurements should be considered for the choice of ROIs. Further research is warranted regarding the value of IVIM MR imaging in the diagnosis and staging of CLD and LC. 


\section{Data Availability}

The datasets generated during the current study are available from the corresponding author on reasonable request.

\section{Disclosure}

Lianxiang Xiao is the co-first author.

\section{Conflicts of Interest}

The authors declare that they have no conflict of interest.

\section{References}

[1] J. Bruix, G. J. Gores, and V. Mazzaferro, "Hepatocellular carcinoma: clinical frontiers and perspectives," Gut, vol. 63, no. 5, pp. 844-855, 2014.

[2] J. Bruix and M. Sherman, "Management of hepatocellular carcinoma: an update," Hepatology, vol. 53, no. 3, pp. 1020-1022, 2011.

[3] D. E. Kaplan, F. Dai, M. Skanderson et al., "Recalibrating the child-Turcotte-Pugh score to improve prediction of transplant-free survival in patients with cirrhosis," Digestive Diseases and Sciences, vol. 61, no. 11, pp. 3309-3320, 2016.

[4] A. Luciani, A. Vignaud, M. Cavet et al., "Liver cirrhosis: intravoxel incoherent motion MR imaging-pilot study," Radiology, vol. 249, no. 3, pp. 891-899, 2008.

[5] H. Dijkstra, P. Baron, P. Kappert, M. Oudkerk, and P. E. Sijens, "Effects of microperfusion in hepatic diffusion weighted imaging," European Radiology, vol. 22, no. 4, pp. 891-899, 2012.

[6] H. A. Dyvorne, N. Galea, T. Nevers et al., "Diffusion-weighted imaging of the liver with multiple b values: effect of diffusion gradient polarity and breathing acquisition on image quality and intravoxel incoherent motion parameters-a pilot study," Radiology, vol. 266, no. 3, pp. 920-929, 2013.

[7] B. M. A. Delattre, M. Viallon, H. Wei et al., "In vivo cardiac diffusion-weighted magnetic resonance imaging: quantification of normal perfusion and diffusion coefficients with intravoxel incoherent motion imaging," Investigative Radiology, vol. 47, no. 11, pp. 662-670, 2012.

[8] J. H. Yoon, J. M. Lee, J. H. Baek et al., "Evaluation of hepatic fibrosis using intravoxel incoherent motion in diffusionweighted liver MRI," Journal of Computer Assisted Tomography, vol. 38, no. 1, pp. 110-116, 2014.

[9] D. Le Bihan and R. Turner, "The capillary network: a link between IVIM and classical perfusion," Magnetic Resonance in Medicine, vol. 27, no. 1, pp. 171-178, 1992.

[10] S. Woo, J. M. Lee, J. H. Yoon, I. Joo, J. K. Han, and B. I. Choi, "Intravoxel incoherent motion diffusion-weighted MR imaging of hepatocellular carcinoma: correlation with enhancement degree and histologic grade," Radiology, vol. 270, no. 3, pp. 758-767, 2014.

[11] J. Patel, E. E. Sigmund, H. Rusinek, M. Oei, J. S. Babb, and B. Taouli, "Diagnosis of cirrhosis with intravoxel incoherent motion diffusion MRI and dynamic contrast-enhanced MRI alone and in combination: preliminary experience," Journal of Magnetic Resonance Imaging, vol. 31, no. 3, pp. 589-600, 2010.

[12] D. M. Koh, D. J. Collins, and M. R. Orton, "Intravoxel incoherent motion in body diffusion-weighted MRI: reality and challenges," American Journal of Roentgenology, vol. 196, no. 6, pp. 1351-1361, 2011.

[13] A. E. Samir, M. Dhyani, A. Vij et al., "Shear-wave elastography for the estimation of liver fibrosis in chronic liver disease: determining accuracy and ideal site for measurement," Radiology, vol. 274, no. 3, pp. 888-896, 2015.

[14] C. H. Wu, M. C. Ho, Y. M. Jeng et al., "Assessing hepatic fibrosis: comparing the intravoxel incoherent motion in MRI with acoustic radiation force impulse imaging in US," European Radiology, vol. 25, no. 12, pp. 3552-3559, 2015.

[15] D. B. Parente, F. F. Paiva, J. A. Oliveira Neto et al., "Intravoxel incoherent motion diffusion weighted $\mathrm{MR}$ imaging at $3.0 \mathrm{~T}$ : assessment of steatohepatitis and fibrosis compared with liver biopsy in type 2 diabetic patients," PLoS One, vol. 10, no. 5, article e0125653, 2015.

[16] S. R. Chung, S. S. Lee, N. Kim et al., "Intravoxel incoherent motion MRI for liver fibrosis assessment: a pilot study," Acta Radiologica, vol. 56, no. 12, pp. 1428-1436, 2015.

[17] J. P. Cercueil, J. M. Petit, S. Nougaret et al., "Intravoxel incoherent motion diffusion-weighted imaging in the liver: comparison of mono-, bi- and tri-exponential modelling at 3.0-T," European Radiology, vol. 25, no. 6, pp. 1541-1550, 2015.

[18] A. M. Chow, D. S. Gao, S. J. Fan et al., "Liver fibrosis: an intravoxel incoherent motion (IVIM) study," Journal of Magnetic Resonance Imaging, vol. 36, no. 1, pp. 159-167, 2012.

[19] W. W. Lautt, "Hepatic vasculature: a conceptual review," Gastroenterology, vol. 73, no. 5, pp. 1163-1169, 1977.

[20] B. E. Van Beers, I. Leconte, R. Materne, A. M. Smith, J. Jamart, and Y. Horsmans, "Hepatic perfusion parameters in chronic liver disease: dynamic CT measurements correlated with disease severity," American Journal of Roentgenology, vol. 176, no. 3, pp. 667-673, 2001.

[21] K. Hashimoto, T. Murakami, K. Dono et al., "Assessment of the severity of liver disease and fibrotic change: the usefulness of hepatic CT perfusion imaging," Oncology Reports, vol. 16, no. 4, pp. 677-683, 2006.

[22] S. Tutcu, S. Serter, Y. Kaya et al., "Hepatic perfusion changes in an experimental model of acute pancreatitis: evaluation by perfusion CT," European Journal of Radiology, vol. 75, no. 2, pp. 203-206, 2010.

[23] B. Y. Su, Z. Y. Jin, W. Liu et al., "Features of eight segments of liver perfusion with the second generation dual-source computed tomography," Zhongguo Yi Xue Ke Xue Yuan Xue Bao, vol. 32, no. 6, pp. 655-658, 2010.

[24] S. J. Hectors, M. Wagner, C. Besa et al., "Intravoxel incoherent motion diffusion-weighted imaging of hepatocellular carcinoma: is there a correlation with flow and perfusion metrics obtained with dynamic contrast-enhanced MRI?," Journal of Magnetic Resonance Imaging, vol. 44, no. 4, pp. 856-864, 2016.

[25] M. Wang, X. Li, J. Zou, X. Chen, S. Chen, and W. Xiang, "Evaluation of hepatic tumors using intravoxel incoherent motion diffusion-weighted MRI," Medical Science Monitor, vol. 22, pp. 702-709, 2016.

[26] M. Lewin, A. Poujol-Robert, P. Y. Boelle et al., "Diffusionweighted magnetic resonance imaging for the assessment of fibrosis in chronic hepatitis C," Hepatology, vol. 46, no. 3, pp. 658-665, 2007.

[27] B. Taouli, M. Chouli, A. J. Martin, A. Qayyum, F. V. Coakley, and V. Vilgrain, "Chronic hepatitis: role of diffusion-weighted 
imaging and diffusion tensor imaging for the diagnosis of liver fibrosis and inflammation," Journal of Magnetic Resonance Imaging, vol. 28, no. 1, pp. 89-95, 2008.

[28] B. Taouli, A. J. Tolia, M. Losada et al., "Diffusion-weighted MRI for quantification of liver fibrosis: preliminary experience," American Journal of Roentgenology, vol. 189, no. 4, pp. 799-806, 2007.

[29] K. Sandrasegaran, F. M. Akisik, C. Lin et al., "Value of diffusion-weighted MRI for assessing liver fibrosis and cirrhosis," American Journal of Roentgenology, vol. 193, no. 6, pp. 1556-1560, 2009.

[30] A. A. Bakan, E. Inci, S. Bakan, S. Gokturk, and T. Cimilli, "Utility of diffusion-weighted imaging in the evaluation of liver fibrosis," European Radiology, vol. 22, no. 3, pp. 682-687, 2012.

[31] Q. B. Wang, H. Zhu, H. L. Liu, and B. Zhang, "Performance of magnetic resonance elastography and diffusion-weighted imaging for the staging of hepatic fibrosis: a meta-analysis," Hepatology, vol. 56, no. 1, pp. 239-247, 2012.

[32] M. Tosun, N. Inan, H. T. Sarisoy et al., "Diagnostic performance of conventional diffusion weighted imaging and diffusion tensor imaging for the liver fibrosis and inflammation," European Journal of Radiology, vol. 82, no. 2, pp. 203-207, 2013.

[33] K. Fujimoto, T. Tonan, S. Azuma et al., "Evaluation of the mean and entropy of apparent diffusion coefficient values in chronic hepatitis C: correlation with pathologic fibrosis stage and inflammatory activity grade," Radiology, vol. 258, no. 3, pp. 739-748, 2011.

[34] J. Zhang, Y. Guo, X. Tan et al., "MRI-based estimation of liver function by intravoxel incoherent motion diffusion-weighted imaging," Magnetic Resonance Imaging, vol. 34, no. 8, pp. 1220-1225, 2016.

[35] M. Franca, L. Marti-Bonmati, A. Alberich-Bayarri et al., "Evaluation of fibrosis and inflammation in diffuse liver diseases using intravoxel incoherent motion diffusion-weighted MR imaging," Abdominal Radiology, vol. 42, no. 2, pp. 468-477, 2017.

[36] A. Muhler, I. Heinzelmann, and H.-J. Weinmann, "Elimination of gadolinium-ethoxybenzyl-DTPA in a rat model of severely impaired liver and kidney excretory function, an experimental study in rats," Investigative Radiology, vol. 29, no. 2, pp. 213-216, 1994.

[37] E. Talakic, J. Steiner, P. Kalmar et al., "Gd-EOB-DTPA enhanced MRI of the liver: correlation of relative hepatic enhancement, relative renal enhancement, and liver to kidneys enhancement ratio with serum hepatic enzyme levels and eGFR," European Journal of Radiology, vol. 83, no. 4, pp. 607-611, 2014.

[38] A. Andreou, D. M. Koh, D. J. Collins et al., "Measurement reproducibility of perfusion fraction and pseudodiffusion coefficient derived by intravoxel incoherent motion diffusion-weighted MR imaging in normal liver and metastases," European Radiology, vol. 23, no. 2, pp. 428-434, 2013.

[39] T. Hayashi, T. Miyati, J. Takahashi et al., "Diffusion analysis with triexponential function in liver cirrhosis," Journal of Magnetic Resonance Imaging, vol. 38, no. 1, pp. 148-153, 2013.
[40] A. Lemke, F. B. Laun, D. Simon, B. Stieltjes, and L. R. Schad, "An in vivo verification of the intravoxel incoherent motion effect in diffusion-weighted imaging of the abdomen," Magnetic Resonance in Medicine, vol. 64, no. 6, pp. 1580-1585, 2010.

[41] A. Lemke, B. Stieltjes, L. R. Schad, and F. B. Laun, “Toward an optimal distribution of $\mathrm{b}$ values for intravoxel incoherent motion imaging," Magnetic Resonance Imaging, vol. 29, no. 6, pp. 766-776, 2011. 


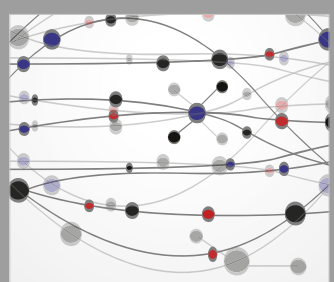

The Scientific World Journal
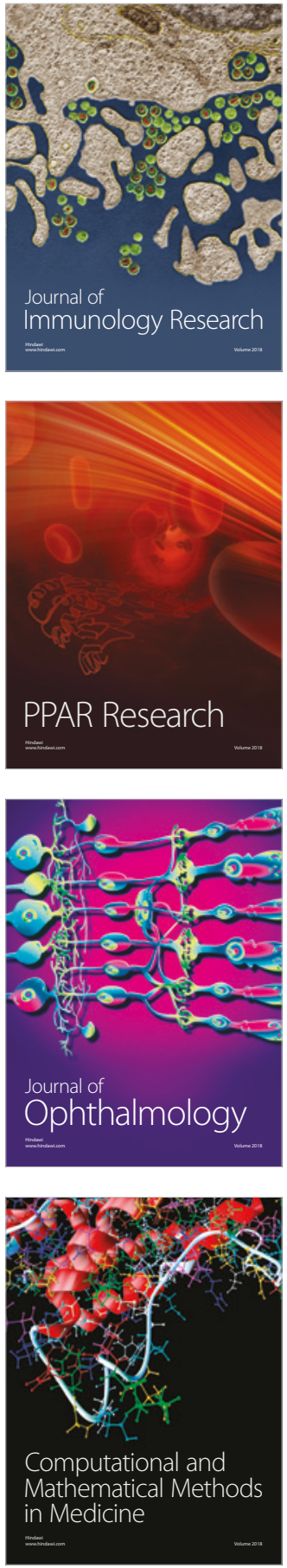

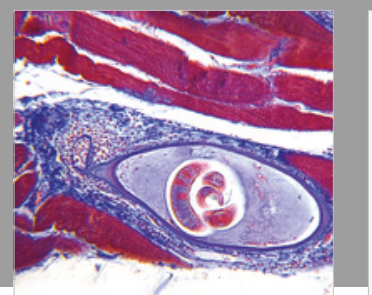

Gastroenterology Research and Practice

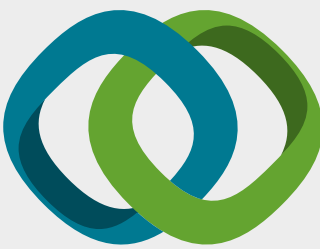

\section{Hindawi}

Submit your manuscripts at

www.hindawi.com
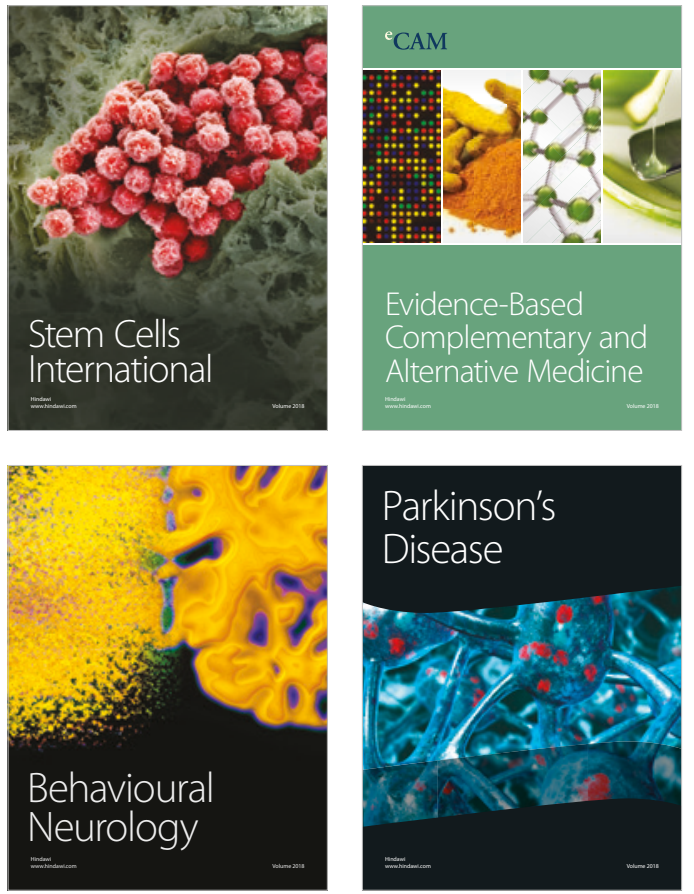

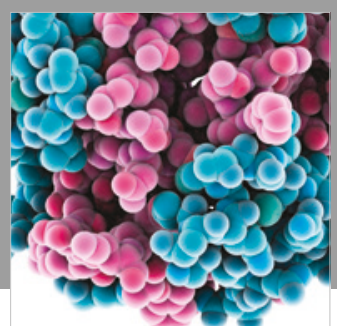

ournal of

Diabetes Research

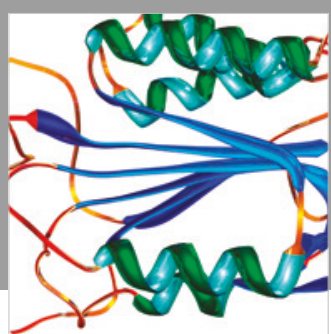

Disease Markers
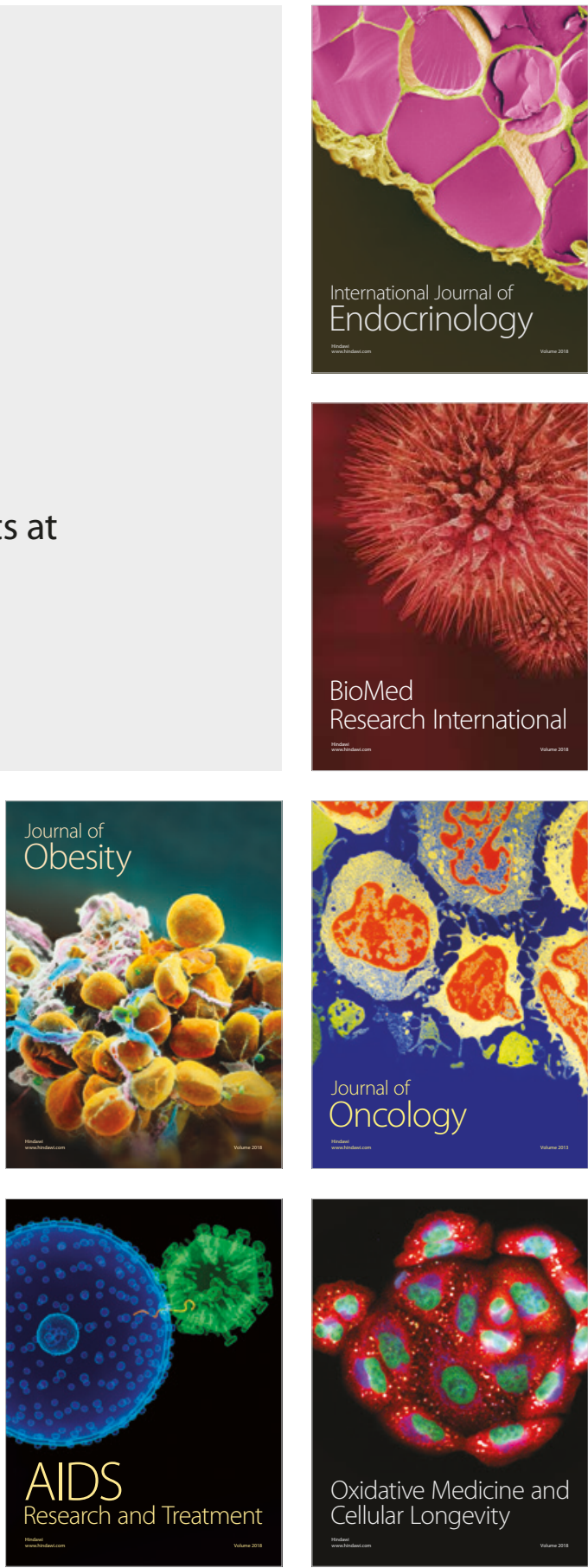